ice core, in which the entire transition $(120,000$ to $60,000 \mathrm{yr} \mathrm{BP}$ ?) from the last and really warm interglacial (the Eem) to full glacial conditions is revealed by oxygen isotope ratio changes, would probably lead to the long-needed climatic counterpart to the continental ice volume records derived from analyses of foraminifera in deep-sea cores $^{17-19}$.

These records show that at the end of the Eem some 120,000 yr ago the amount of continental ice began to increase, and that it grew from less than today to more than double the present amount within less than $10,000 \mathrm{yr}$. The drastic climatic deterioration, which triggered off the enormous glacier advance, may have taken place within a much shorter time interval ${ }^{20}$, perhaps a century or less, to judge from a Greenland isotope record ${ }^{21}$ and from pollen records ${ }^{22-24}$. However, these records are poorly dated before $50,000 \mathrm{BP}$, and the possibility of a discontinuity in the layer sequences must be taken into account, the more so as the suggested duration (one millenium only) of the cold period that succeeded the Eem is inconsistent with the build-up of huge amounts of continental ice.

It is extremely important to study why and how abruptly the Eemian interglacial came to an end, because it might change our conception of atmospheric stability, and describe a possible mode of termination of the present interglacial. The new radioisotope ice dating technique will not be able to reveal the duration of the past climatic shift, but a counting of annual layers, deposited in a continuous sequence throughout the shift, may be feasible by modified detection techniques, because such layers, each $1 \mathrm{~mm}$ thick, probably exist some $80 \mathrm{~m}$ above the bottom of the ice sheet in central Greenland ${ }^{16}$.

1. Oeschger, H. Proc. Symp chem and radioact. Dating Am. Chem. Soc., Houston, Texas (March 1980).

Delmas, R. . Ascencio, J.M. \& Legrand, M. Nature 284, 155(1980)

3. Johnsen, S.J., Dansgaard, W., Clausen, H.B.\& Langway, C.C. Naiure 235,429 (1972)

4. Dansgaard, W. et al. Nature 255, 24 (1975)

5. Lorius, C., Merlivat, L., Jouzel, J.\& Pourchet, M. Nature 280, 644 (1979).

6. Reeh, N. et al. J. Glaciol. 20, 27 (1978)

7. Herron, M.M., Langway, C.C., Weiss, H.V. \& Cragin, I. H. Geochim. cosmochim. Acta 41, 915 (1977)

8. Hammer, C.U., Clausen, H.B. \& Dansgaard, W. Nature 288,230 (1980).

9. Barkov, N.I. US Antarc. J. 10, No. 2, 55 (1975)

10. Hammer, C.U. et al. J. Glaciol. 20, 3 (1978).

11. Oeschger, H., Stauffer, B., Brucher, P. \& Moell, M. J. Glaciol. 17, 117 (1976).

12. Proc. Ist Conf. on Radiocarbon Dating with Accelerators Univ. Rochester (ed. Gove, H.E.) (1978).

3. Raisbeck, G.M., Yiou, F., Fruneau, M., Lieuvin, M. \& Raisbeck, G.M., Yiou, F., Fruneau,

Loiseaux, J.M. Nature 275, 731 (1978).
Bennett, C. I. et al. Science 201, 345 (1978).

14. Bennett, C.L.. et al. Science 201, 345 (1978).

5. Finkel, R.C., Nishiizumi, K., Elmore, D., Ferraro, R.D. \& Gove, H.E. Geophys. Res. Lett. 7, 983 (1980)

16. Dansgaard, W., Johnsen, S.J., Clausen, H.B. \& Gundestrup, N. Meddeletser om Gronland 197, No. 2, (1973).

17. Emiliani, C. J. Geol. 74, 109 (1966).

18. Hays, J.D., Imbrie, J. \& Shackleton, N.J. Science 194, 1121 (1976).

19. Duplessy, J.-C. in Climatic Change (ed. Gribbin, J.) 46 (Cambridge Univ. Press, 1978).

20. Dansgaard, W. \& Duplessy, J.-C. Boreas 10, No. 2 (1981).

21. Dansgaard, W., Johnsen, S.J., Clausen. H.B. \& I angway, C.C. Quat. Res. 2, 296 (1972).

22. Andersen, S.T. Medd. Dansk Geol. Foren. 15, 90 (1969).

23. Wijmstra, T.A. Acta bot, neerl. 18, 511 (1969).

24. Woillard, G. Quat. Res. 9, 1 (1978).

\title{
The origin of cosmic gamma-ray bursts - new Soviet results
}

\section{From B. J. Teegarden}

SINCE their accidental discovery in 1973 by Vela spacecraft, gamma-ray bursts have continued to be a mysterious and intriguing phenomenon. Lasting from a few seconds to a few tens of seconds, these bright flashes of gamma radiation occur randomly every one to two weeks. With one possible exception the identification of the sources of these events remains unsolved.

A multi-spacecraft network including experiments designed in the United States, France and the Soviet Union has been used to time the arrival of the gamma-ray burst wavefront to determine the arrival direction of the radiation. The only burst for which a source identification was made is the very unusual event of 5 March 1979 which displayed a very sharp, intense radiation spike lasting only 0.1 second and was followed by 8 second oscillations lasting for approximately 100 seconds The arrival direction of this burst coincided with the remnant of a supernova explosion (labelled N49) in the Large Magellanic Cloud, our nearest neighbouring galaxy. This, however, was a highly unusual event, and its identification is still not universally accepted. At this time no other gamma-ray burst source identifications have been made. This is in spite of the fact that arrival directions for a number of events have been precisely determined.

A number of different models have been put forth to explain the energy source of these bursts. Two of these may be briefly summarized: the first hypothesizes that a solid body falls onto the surface of a neutron star providing a nearly instantaneous release of energy. The second proposes that infalling material from either a companion star or the interstellar medium gradually builds up a surface layer at the poles of a neutron star. Sufficient accumulation of material over a period of time leads to an unstable condition wherein a thermonuclear explosion can occur. In either case the resultant heating of the atmosphere immediately above the surface of the neutron star is sufficient to cause the release of an intense burst of gamma radiation.

Some dramatic new observations of gamma-ray bursts have recently been reported by E. P. Mazets and his colleagues at the Ioffe Physical-Technical Institute in Leningrad (see this issue of Nature, p.378) that shed important new light on the question of the origin of these events. Mazets and his co-workers have measured

B. J. Teegarden is in the Laboratory for High Energy Astrophysics NASA/Goddard Space Flight Center. the spectra of a large number of bursts using detectors on board the Soviet Venera 11 and 12 spacecraft. They have found remarkable emission and absorption features in 26 of the 80-90 events that were measured. Most of the spectra display pronounced absorption in the $30-70 \mathrm{keV}$ range. This has been intepreted as being due to a phenomenon known as cyclotron absorption.

It is expected that extremely strong magnetic fields will surround a neutron star. This follows if the magnetic flux is conserved during the collapse of the parent star down to the neutron star. A simple extrapolation from the parent star to its compact offspring yields a magnetic field in the $10^{12}$ gauss range, a value far beyond any achieved in the laboratory. An electron in such a field will be constrained to spiral tightly about the magnetic field line. Quantum theory predicts that the electrons will be confined to discrete energy levels and that the transitions between these levels will be accompanied by either the emission or absorption of radiation. In a $5 \times 10^{12}$ gauss magnetic field the characteristic energy of the transition is $\sim 50 \mathrm{keV}$. The absorption and emission features in gamma-ray burst spectra observed by Mazets and his colleagues are therefore consistent with cyclotron processes in the strong magnetic fields expected to exist near the surface of a neutron star.

In addition to the 'cyclotron' emission and absorption features, Mazets and coworkers, as well as a group at Goddard Space Flight Center in the US, have seen in a smaller group of events (about 6 ) a broad emission line in the $400-450 \mathrm{keV}$ range. When a positron and electron encounter each other and annihilate, they give off a characteristic emission at $511 \mathrm{keV}$ known as annihilation radiation. Any such radiation originating near the surface of a neutron star will lose energy as it escapes from this intense gravitational field. This energy change is known as a 'gravitational redshift'. Present models predict that the magnitude of this shift will be $15-20 \%$ for a neutron star whose mass is the same as the Sun. Any $511 \mathrm{keV}$ annihilation radiation produced near a neutron star surface would, therefore, emerge from the star's gravitational field with an energy in the $400-450 \mathrm{keV}$ range.

It should be emphasized that these results are very recent and that the interpretations discussed here are not necessarily unique. These Soviet results, however, seem to point very strongly towards a neutron star origin for cosmic gammy-ray bursts. 